\title{
Online Electron/Jet Neural High-level Trigger Over Independent Calorimetry Information
}

\author{
Rodrigo Coura Torres* \\ Federal University of Rio de Janeiro / CERN \\ E-mail: Rodrigo. Torres@cern.ch \\ José Manoel Seixas \\ Federal University of Rio de Janeiro \\ E-mail: seixaselps.ufrj.br
}

\section{Andre Rabello dos Anjos \\ University of Wisconsin, Madison \\ E-mail: Andre.dos. Anjosecern. ch}

\section{Danilo Vannier Cunha}

Federal University of Rio de Janeiro / CERN

E-mail: dcunha@lps.ufrj.br

\begin{abstract}
A data volume of $60 \mathrm{~TB} / \mathrm{s}$ is expected from the high LHC collision rate and high resolution of the ATLAS detectors. To cope with this bandwidth, a highly programmable, three-level online triggering system is under development. One of the main components of this system is an electron/jet discriminator that uses the highly segmented calorimetry information. In this work, we address the electron/jet discrimination at the second-level trigger by building a set of concentric ring sums around the energy deposition peak in each calorimeter segment. An Independent Component Analysis (ICA) on these ring sums is then performed to extract the main sources of the calorimeter signal. The extracted independent components feed the input nodes of a neural electron/jet discriminator. The proposed system is able to achieve higher detection efficiency than the current electron/jet discriminating system operating in ATLAS, while being fast enough to cope with the time restrictions of the ATLAS triggering system operation.
\end{abstract}

XI International Workshop on Advanced Computing and Analysis Techniques in Physics Research April 23-27 2007

Amsterdam, the Netherlands

\footnotetext{
* Speaker.
} 


\section{Introduction}

High energy physics is known to be a highly challenging research field, demanding state of the art technologies [1]. In this field, filtering systems are intensively used, specially in modern particle collider experiments, due to the high number of events generated, together with the typical very low rate of interesting events. Also, in this kind of experiments, it is common to have a mixture of information, in such a way that the rare event of interest may be missed due to its combination with a masking background noise while interacting with the detectors [2]. Therefore, if one could separate the information regarding the signal sources, the discriminating system could profit from this, being able to restrict its analysis to the information that is useful to the discrimination process. This would allow optimal signal compaction (with direct impact on system execution time and complexity) as well as high detection efficiency.

Presently, at CERN, LHC (Large Hadron Collider) [3], the new generation particle accelerator, is being prepared for start operation by the year 2008. This accelerator has $27 \mathrm{~km}$ extension, and it is capable of colliding particles at a rate of $40 \mathrm{MHz}$, with $14 \mathrm{Tev}$ in their center of mass. ATLAS (A Toroidal LHC Apparatus) [4] is one of the main detection laboratories to be placed around the collision points for analyzing LHC data. ATLAS is made of different highly segmented subdetectors (see Fig. 1), which produce high resolution measurements. As a result of both detector granularity and LHC collision rate, it is expected a data stream of $60 \mathrm{~TB} / \mathrm{s}$. However, the event of interest may occur with a frequency smaller than $1 \mathrm{~Hz}$. Therefore, ATLAS relies strongly in its online triggering system.

This paper presents an online second-level (LVL2) discriminating system for electron/jet separation using information provided by the calorimetry system of ATLAS. Calorimeters play a major role in modern collider experiments due to their fast response and good energy resolution, as measuring the energy of the incoming particles [5]. At LHC, electrons are messengers of the interesting physics (signal), whereas jets mask the signal signature, acting as background noise.

In ATLAS, the calorimetry system has two sections (electromagnetic and hadronic), and each section is split into layers (seven, in total). Due to the high dimension of the calorimetry data space, the LVL2 triggering system operates on topologically pre-processed data. In our case, data are described by means of summing the layer energy over concentric rings, which are built around the calorimeter cell that samples the maximum energy for a given event. In order to find the independent signal sources, Independent Component Analysis is applied over pre-processed data. The discrimination process is performed on such independent components by using an artificial neural network.

This paper is organized as it follows. Section 2 briefly describes ATLAS and its triggering system. Next, Section 3 introduces the basic concepts of independent component analysis. Then, Section 4 describes the topological pre-processing method and Section 5 presents the independent components applied over the generated ring sums. Later, in Section 6, results are presented for the proposed discriminating system. Finally, Section 7 derives some conclusions.

\section{The ATLAS Project}

The ATLAS project is a worldwide collaboration of 36 countries, and more than 1000 insti- 


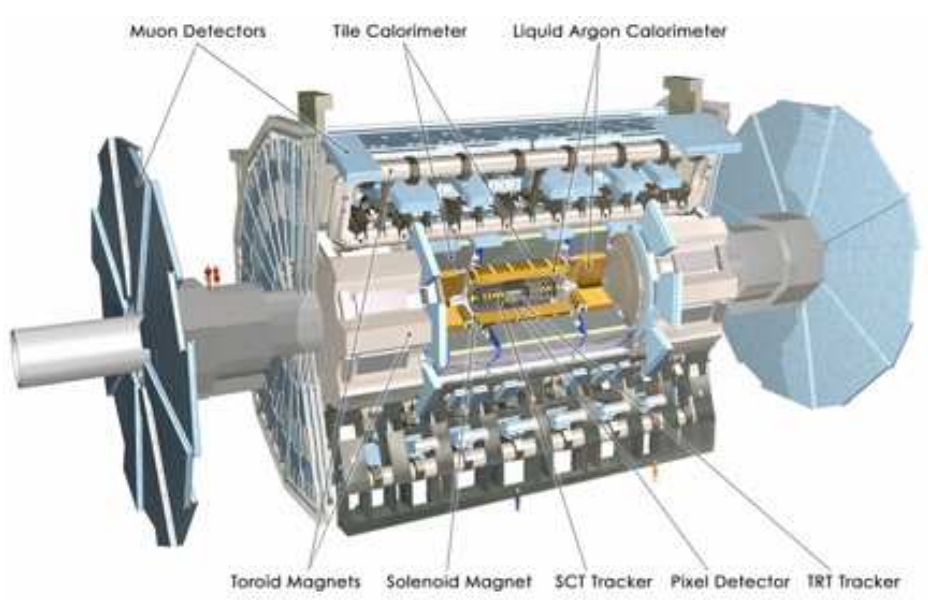

Figure 1: The ATLAS detector diagram.

tutes. The main goal of this collaboration is to experimentally prove the existence of the Higgs Boson [6]. To cope with the many sub-products obtained in proton-proton collisions, the ATLAS is composed by many sub-detectors, as it can be seen in Fig. 1. First we have the particle tracking section. The solenoid magnets provide the electromagnetic field so that, together with the TRT and SCT trackers, and the Pixel Detector, the trajectory of low momentum particles can be calculated. For muons, due to their high momentum, a special tracking system was developed. Enormous toroidal magnets provide the magnetic field required by the muon detector. Next, we have the calorimetry system, which comprises two sections:

1. Electromagnetic Calorimeter (Liquid Argon Calorimeter): measures the energy of electrons and photons. This calorimeter is divided in 4 layers, with distinct thickness (third layer is the thickest). Besides, each layer has different levels of granularity, which may even vary within a layer. The resolution of each section of the electromagnetic calorimeter can be observed in Tab. 1.

2. Hadronic Calorimeter (Tile Calorimeter): absorbs the energy of hadrons. This calorimeter is also divided in three layers of energy deposition, with variable granularity and thickness. The resolution of each section of the hadronic calorimeter can be observed in Tab. 2 .

By looking at the way objects normally interact with a calorimeter, it is possible to determine which kind of particle they are. Fig. 2 displays the interaction of a typical electron with the various calorimeter layers.

\subsection{Online Events Selection}

To cope with LHC stringent conditions, an online triggering system is being developed [7], [8]. This triggering system will be responsible for selecting the interesting events among the mass of background noise, reducing the amount of stored data. The triggering system comprises three sequential levels. 
Table 1: Segmentation of each layer of the e.m. calorimeter.

\begin{tabular}{|c|c|c|c|c|}
\hline Layer & Part & $\eta_{\text {start }}$ & $\eta_{\text {end }}$ & $\Delta \eta \times \Delta \phi$ \\
\hline \multirow{2}{*}{$\begin{array}{l}\text { Pre- } \\
\text { sampler }\end{array}$} & Barrel & 0 & 1.5 & $0.025 \times 0.1$ \\
\hline & Endcap & 1.5 & 1.8 & $0.025 \times 0.1$ \\
\hline \multirow{7}{*}{ Layer 1} & Barrel & $\begin{array}{c}0 \\
1.4\end{array}$ & $\begin{array}{c}1.4 \\
1.475\end{array}$ & $\begin{array}{l}0.003125 \times 1 \\
0.025 \times 0.025\end{array}$ \\
\hline & \multirow{6}{*}{ Endcap } & 1.375 & 1.5 & $0.025 \times 0.1$ \\
\hline & & 1.5 & 1.8 & $0.003125 \times 0.1$ \\
\hline & & 1.8 & 2.0 & $0.004167 \times 0.1$ \\
\hline & & 2.0 & 2.4 & $0.00625 \times 0.1$ \\
\hline & & 2.4 & 2.5 & $0.025 \times 0.1$ \\
\hline & & 2.5 & 3.2 & $0.1 \times 0.1$ \\
\hline \multirow{4}{*}{ Layer 2} & \multirow{2}{*}{ Barrel } & 0 & 1.4 & $0.025 \times 0.025$ \\
\hline & & 1.4 & 1.475 & $0.075 \times 0.025$ \\
\hline & \multirow{2}{*}{ Endcap } & 1.375 & 2.5 & $0.025 \times 0.025$ \\
\hline & & 2.5 & 3.2 & $0.1 \times 0.1$ \\
\hline \multirow{2}{*}{ Layer 3} & Barrel & 0 & 1.35 & $0.05 \times 0.025$ \\
\hline & Endcap & 1.5 & 2.5 & $0.05 \times 0.025$ \\
\hline
\end{tabular}

Table 2: Segmentation of each layer of the hadronic calorimeter.

\begin{tabular}{||l|l|c|c|c|}
\hline Layer & Part & $\eta_{\text {start }}$ & $\eta_{\text {end }}$ & $\Delta \eta \times \Delta \phi$ \\
\hline \hline \multirow{3}{*}{ Layers 1 \& } & Barrel (TileCal) & 0 & 1.0 & $0.1 \times 0.1$ \\
\cline { 2 - 5 } 2 & Ext. Barrel (TileCal) & 0.8 & 1.7 & $0.1 \times 0.1$ \\
\cline { 2 - 5 } & \multirow{2}{*}{ Endcap (LAr) } & 1.5 & 2.5 & $0.1 \times 0.1$ \\
& & 2.5 & 3.2 & $0.2 \times 0.2$ \\
\hline \multirow{3}{*}{ Layer 3 } & Barrel (TileCal) & 0 & 1.0 & $0.2 \times 0.1$ \\
\cline { 2 - 5 } & Ext. Barrel (TileCal) & 0.8 & 1.7 & $0.2 \times 0.1$ \\
\cline { 2 - 5 } & \multirow{2}{*}{ Endcap (LAr) } & 1.5 & 2.5 & $0.1 \times 0.1$ \\
& & 2.5 & 3.2 & $0.2 \times 0.2$ \\
\hline
\end{tabular}

1. The first-level will produce the highest rejection rate. Due to its severe time conditions ( $2.5 \mu$ s maximum latency per event), this level is implemented in low programability hardware (FPGAs) [9]. Also, calorimeter resolution for this level is reduced, in order to increase data throughput. So, this level will reject only events with well distinctive features, by looking to the calorimeters and the muon detector only. It is expected that this level will reduce the number of events up to approximately $75 \mathrm{kHz}$. Also, this level is responsible for marking the regions in the detector where an approved event was found. This regions are called Regions of Interest (RoI) and only these regions are propagated to the second-level, in order to increase the system bandwidth [7].

2. The second-level will receive the RoIs marked by the first-level, and will validate the decision of the previous level by using full detector information for each RoI, analyzing information coming from the calorimeters and tracking system. Since this level has no more than 10 ms of latency, a multiprocessing environment comprising 500 dual-core personal computers (PC) is envisaged. In this level, optimized algorithms written in high level programming 


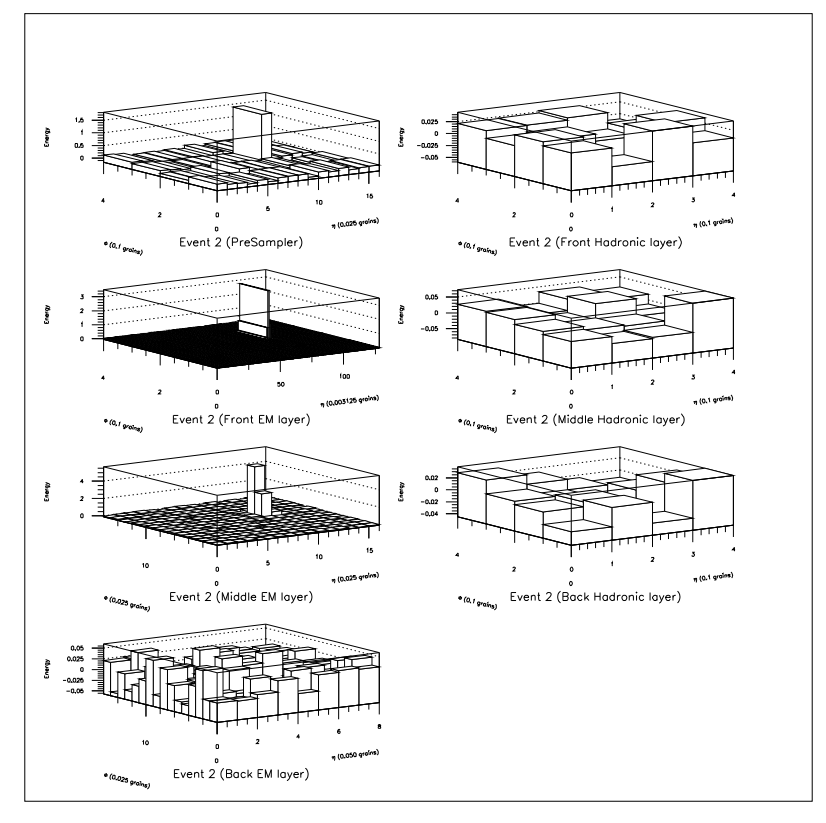

Figure 2: An electron interacting with the ATLAS calorimeter.

language $(\mathrm{C}++)$ are being developed. At the second-level output, the event rate should be reduced to approximately $2 \mathrm{kHz}$.

3. The third level (also known as Event Filter) will look to the entire detector, in its full granularity, reconstructing particle trajectories, and using all the information available for each event. To accomplish that, the latency time for this level is of the order of one second per event, which also leads to a multiprocessing environment. In this case, approximately 1600 dual-core PCs will be required. Events that pass the Event Filter conditions will be stored in permanent media. Approximately 200 events per second will survive.

The block diagram of such triggering system is shown in Fig. 3. The information generated by the detectors are stored in pipeline memories, while the first-level selects which events will be propagated to the second-level. After that, the accepted events goes through readout drivers and are stored in the readout buffers (ROB). The marked RoI data are requested to the ROBs by the second-level supervisor for signal processing. The approved events, before going to the event filter, are first built in the event builder, and the fully built events are then used by the event filter, which will send the approved events to the data recording service.

It is shown, in Fig. 4 the block diagram for the second-level trigger, since the proposed algorithm was developed to be one of the many signal processing algorithms executed at this level. Once the LVL2 supervisor receives the LVL1 result, it selects a LVL2 processing unit (L2PU) and send to it the received LVL1 result. The L2PU then will call all the signal processing algorithms which will validate the LVL1 result received. The RoI information is requested on-the-fly, via RoB fragments to the ReadOut system. To avoid idle times during data requests, each L2PU runs multiple threads. After the chain of algorithms is performed, the LVL2 result is sent back to the 


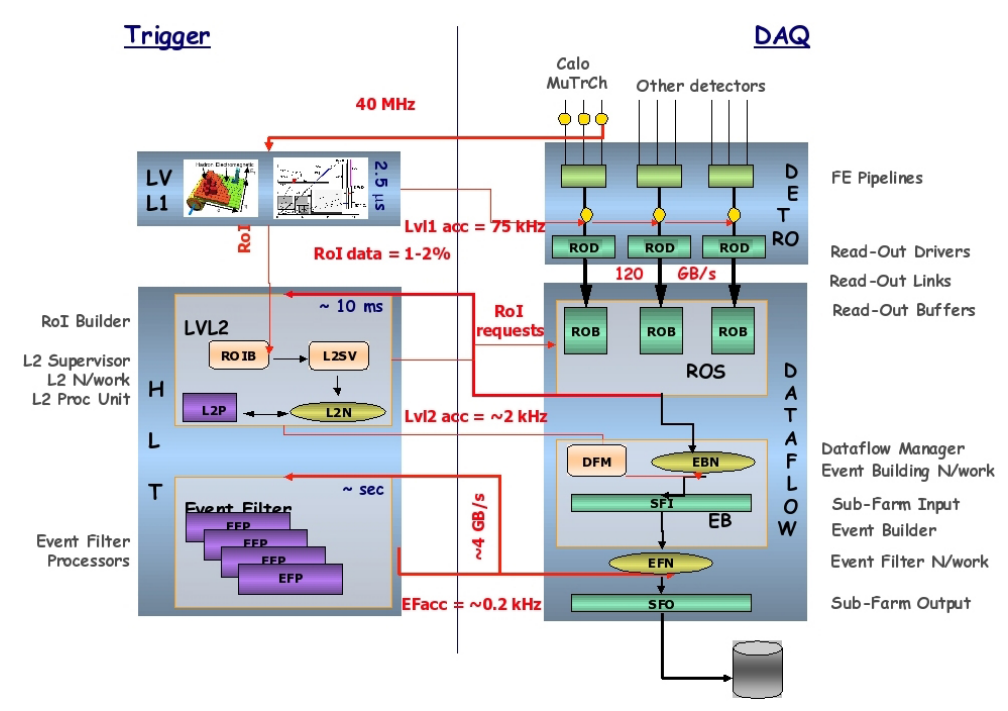

Figure 3: Block diagram of the triggering system.

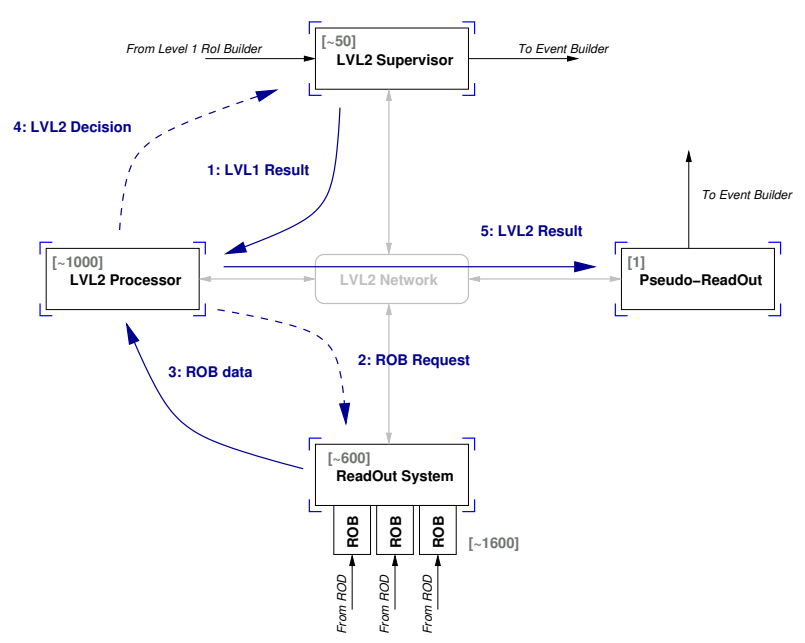

Figure 4: Block diagram of the second-level trigger.

supervisor, which will send it to the Event Builder, which will, either build the full event for the Event Filter if the event was approved, or will simply delete it, in case of LVL2 rejection.

\section{Independent Component Analysis}

In many practical applications, patterns to be discriminated reach the discriminator contaminated either by noise, or by information traces of other independent patterns due to several factors, like detectors characteristics, pile-up effects, etc. This mixture might end up masking relevant patterns, specially if the energy of a desired pattern is small, when compared to the other patterns in the mixture, resulting in loss of detection efficiency of a pattern recognition system. In situations like 
this, it would be nice if the information reaching the discriminator could be separated somehow, so we could isolate each of the independent sources (patterns) within the mixed signal received.

Let's take the input signal $\mathbf{s}=\left[s_{1}, s_{2}, \ldots, s_{N}\right]^{T}$, of size $N$, where $s_{i}$ represents a given incoming independent source. Let's now consider that these independent sources were combined, due to some phenomenon in our detection system, generating the acquired signal $\mathbf{y}$. This could be represented as

$$
\left[\begin{array}{c}
y_{1} \\
y_{2} \\
\vdots \\
y_{N}
\end{array}\right]=\left[\begin{array}{cccc}
m_{11} & m_{12} & \cdots & m_{1 N} \\
m_{21} & m_{22} & \cdots & m_{2 N} \\
\vdots & \vdots & \ddots & \vdots \\
m_{N 1} & m_{N 2} & \cdots & m_{N N}
\end{array}\right] \times\left[\begin{array}{c}
s_{1} \\
s_{2} \\
\vdots \\
s_{N}
\end{array}\right]
$$

where $m_{i j}$ is the weighting factor applied to the $\mathrm{j}$-th source, in order to generated the $\mathrm{i}$-th mixed input. In this manner, Eq. 3.1 can be simplified to

$$
\mathbf{y}=\mathbf{m} \times \mathbf{s}
$$

So, in order to reverse this mixing, and find the independent sources, we must find the matrix $\mathbf{w}=\mathbf{m}^{-1}$, so each independent source vector can be obtained by doing

$$
\mathbf{s}=\mathbf{w} \times \mathbf{y}
$$

where $\mathbf{w}$ now represents the independent components base of our problem. By projecting the vector $\mathbf{y}$ on the independent components matrix (where each row represents an independent component), one can obtain the vector $\mathbf{s}$, which represents the fundamental (unique) information of each source.

The problem, in practice, is how to, having only $\mathbf{y}$, estimate $\mathbf{w}$ ? One good approach for this problem, considering that all sources are statistically independent, is the Central Limit Theorem [10]. Considering

$$
x_{k}=\sum_{i=1}^{k} z_{i}
$$

where $z_{i}$ is an independent and identically distributed random variable. The Central Limit Theorem says that the resulting distribution of the random variable $x_{k}$ will converge to a gaussian distribution with zero mean and unit variance as $k \rightarrow \infty$. Therefore, the resulting distribution of a combination of independent sources will be closer to a gaussian distribution then each distribution individually. Then, if we find the point where each distribution is, as nongaussian as possible, then we can say that we successfully isolated each independent component. There are multiple ways on how to estimate the nongaussianity of a random distribution. One common way to estimate nongaussianity is the kurtosis [10]. For a random variable $y$, It is defined by

$$
\operatorname{kurt}(y)=E\left\{y^{4}\right\}-3\left(E\left\{y^{2}\right\}\right)
$$

The kurtosis carries the property that, as closer to zero it is, the more the distribution can be considered as gaussian. Then finding the maximum nongaussianity between random variables is just a matter of maximizing their kurtosis. Although this approach is very simple to implement, it is not 
optimal when working with acquired data, since the presence of outsider in the distribution might lead to a wrong value of kurtosis, resulting in a wrong independent source estimation.

Another, more robust, way of estimating nongaussianity is using a basic concept from information theory called negentropy [10], or simply entropy. The entropy of a random variable is related to the information that the observation of the variable gives. The more unpredictable a variable is, the larger its entropy. The entropy $H$ of a random vector $\mathbf{y}$, with density $p_{y}(\eta)$ is defined as

$$
H=-\int p_{y}(\eta) \log p_{y}(\eta) d \eta
$$

One fundamental result of information theory is that a gaussian variable has the largest entropy among all random variables of equal variance [10]. Then, to use this concept as a measure of nongaussianity that is zero for a gaussian variable, and always nonnegative, we use the normalized version of differential entropy, given by

$$
J(\mathbf{y})=H\left(\mathbf{y}_{\text {gauss }}\right)-H(\mathbf{y})
$$

where $\mathbf{y}_{\text {gauss }}$ is a gaussian variable of the same correlation (and covariance) matrix as $\mathbf{y}$. In practical problems, we first center and whiten $\mathbf{y}$, so we can set $\mathbf{y}_{\text {gauss }}$ to be a random gaussian variable with zero mean and unity variance. For simplicity, we define, for now on, the vector $\mathbf{z}$ as the vector resulting from the centralization and whitening of $\mathbf{y}$.

To finally estimate our independent sources, we now just need to develop an optimization algorithm that will maximize Eq. 3.7. For that, a fixed point algorithm can be applied. The FastICA [11] finds a direction, i.e., a unit vector $\mathbf{w}$ so that the projection $\mathbf{w}^{T} \mathbf{z}$ maximizes nongaussianity. The FastICA using negentropy combines the superior algorithmic properties resulting from a fixedpoint algorithm, with the statistical properties due to negentropy. There are a few ways of update the value of $\mathbf{w}$ for the FastICA algorithm. For this work, we used the following:

$$
\begin{aligned}
\mathbf{w} & \leftarrow E\left\{\mathbf{z} g\left(\mathbf{w}^{T} \mathbf{z}\right)\right\}-E\left\{g^{\prime}\left(\mathbf{w}^{T} \mathbf{z}\right)\right\} \mathbf{w} \\
g(x) & =x^{3} \\
g^{\prime}(x) & =3 x^{2}
\end{aligned}
$$

Then, by applying iteratively Eqs 3.8-3.10 until stabilization of $\mathbf{w}$, one can find the independent sources for a given problem.

\section{Ring Structure}

The proposed discriminating system is being developed to work within the second-level, using, thus, RoI information [12] as input data. In average, every calorimeter RoI consists up to 1,000 cells spread among different calorimeter layers.

As said in Section 2, one of the main goals of the ATLAS experiment is to find the Higgs Boson. Being the Higgs Boson highly unstable, it soon decays in more stable particles. It is know that one of its possible decaying patterns produces electrons at its final stage, so that the detection 

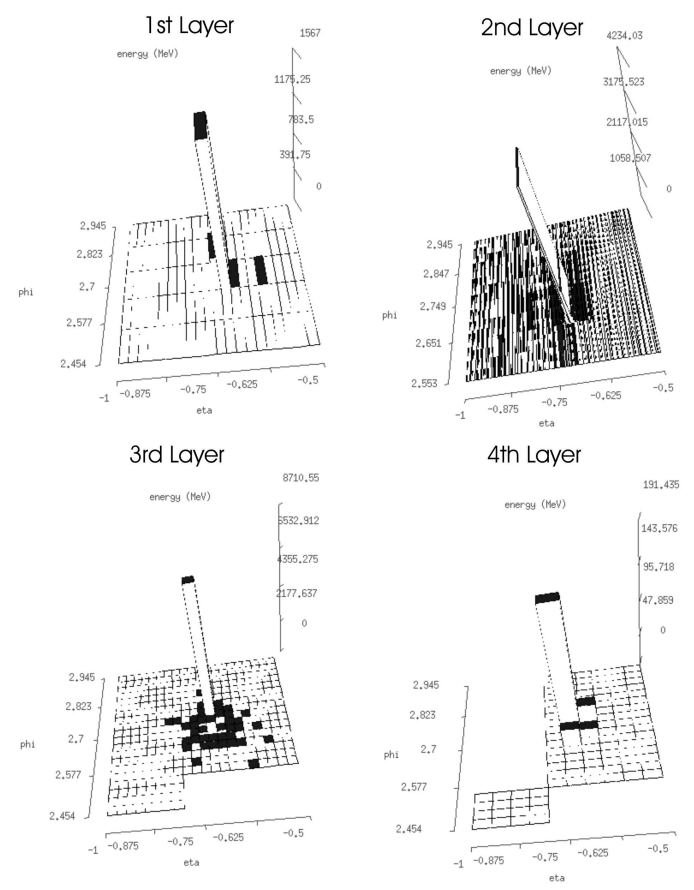

Figure 5: Electron energy deposition on the four layers of the electromagnetic calorimeter.

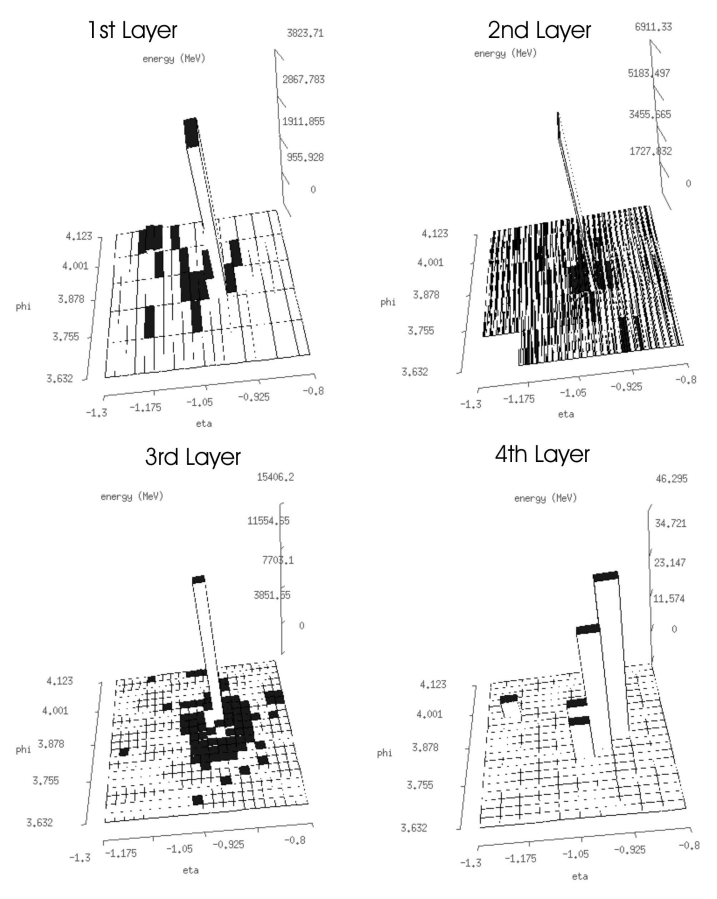

Figure 6: Jet energy deposition on the four layers of the electromagnetic calorimeter. 
of electrons is of great importance for the experiment. Jets, on the other hand, are usually related to background noise, and must be, as early as possible, rejected by the filtering system.

In Figs. 5 and 6 are presented the energy deposition pattern of electrons and jets for the four layers of the electromagnetic calorimeter. The hadronic part, although important from the discrimination point of view, samples little energy for events in the second-level. One can note the subtle differences between the two patterns. It is also possible to verify that jets spread radially their energy while electrons deposit their energy in a more punctual way. So, this analysis shows that the relevant information from the discrimination point of view lies on the surroundings of the hottest cell. Since the radial information is the one which really matter for the discrimination, for each ATLAS calorimeter layer, a set of squared-concentric ring sums is calculated by summing the energy from cells around the central interaction point on that layer [13]. Because the granularity changes radically between the different layers, the number of rings will be different for each layer, as it can be seen in Fig. 7. The width of the rings is determined by the standard layer granularity. Once the rings geometries are calculated, a sum of the cells that fall on those is produced and accumulated. Taking into consideration the standard cell size on these sub-detectors, and an RoI size of 0.4 by 0.4 in $\eta \times \phi, 100$ ring sums are produced for each object tagged by the first-level trigger as electron. The number of generated rings for each layer can be observed in Tab. 3. So, this rings sums can compact the input data space, at the same time that they preserve the physics interpretation of the events, being also easy and fast to implement and execute.

The ring sums, or simply rings, are fed into a normalization system that equalizes the differences in energy of the input objects and better reveals, in a controlled way, the discriminatory variables which are known to be off-center. The normalization is done by

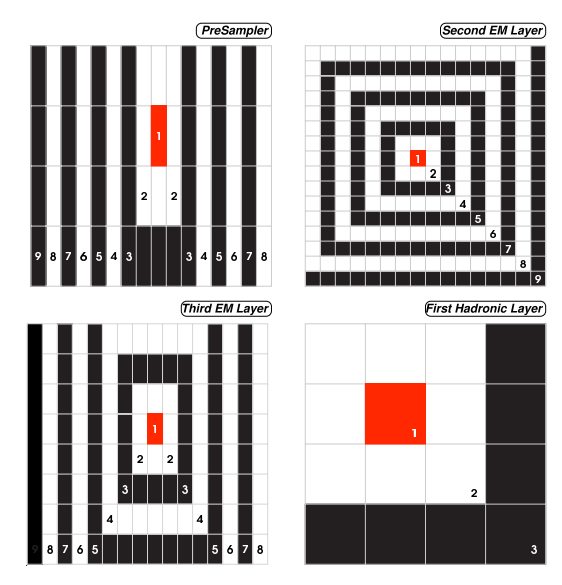

Figure 7: Example of rings produced for each layer.

\begin{tabular}{|l|c|c|c|c|c|c|c|c|}
\hline Layer & $1^{s t}$ & $2^{\text {nd }}$ & $3^{\text {rd }}$ & $4^{\text {th }}$ & $1^{\text {st }}$ & $2^{\text {nd }}$ & $3^{\text {rd }}$ & Total \\
\hline Type & EM & EM & EM & EM & HAD & HAD & HAD & - \\
\hline Rings & 8 & 64 & 8 & 8 & 4 & 4 & 4 & 100 \\
\hline
\end{tabular}

Table 3: Number of generated rings for each detector layer. 


$$
E_{N i}=\frac{E_{i}}{E_{t o t}-\sum_{j=1}^{i-1} E_{j}}
$$

where $E_{i}$ is the original energy of each ring, $E_{t o t}$ is the total layer energy, prior to the normalization. The normalization continues recursively, until some limit on the layer, or the algorithm would be amplifying too much the peripheral noise in the RoI. From this point on, the ring values are divided by a constant [13].

\section{Independent Component Over Ring Sums}

It is known [5] that the energy information of a calorimetry cell is dependent on the surrounding cells, once they are excited by the same particle shower (either electromagnetic or hadronic). Grouping the cells in ring sums, as seen in Section 4 , we produce the input signal $\mathbf{y}=\left[y_{1}, y_{2}, \ldots, y_{N}\right]^{T}$, of size $N$, where $y_{i}$ represents the accumulated energy over ring $i$. After the ringing process, it is desired to find the fundamental (independent) sources which, after being combined, gave place to the event $\mathbf{y}$. Therefore, one can imagine that each ring received the weighted sum of the $N$ independent sources ${ }^{1}$, as seen in Section 3. For our particular case, we consider $m_{i j}$ as the weighting factor applied to the $\mathrm{j}$-th source connected to the $\mathrm{i}$-th sensor (ring).

For our discriminating system, ICA can be used to isolate incoming signal sources from noise and pile-up [2], making the pattern more evident to the hypothesis making algorithm. Fig. 8 presents a typical electron and jet, after the ringing process took place ${ }^{2}$ without IC projection and also after being projected onto their independent components. For this later case, multiples peaks appear for the jet, which is understandable, due to the spreading characteristic of this kind of particle, when compared to electrons, showing that independent components were able to identify and emphasize the distinctions between both particles, which will help the hypothesis making section of the proposed system, as we will see next.

\section{System Development and Results}

The discriminating system was developed using, as input data, simulated RoI data, as it will be seen by the second-level (thus, after a simulation of the level one operation). The classifier used was a feed-forward neural network with one hidden layer. The input layer had 100 nodes and the output layer only one node ( with outputs -1 for electrons and +1 for jets). The training algorithm

\begin{tabular}{|l|c|c|c|}
\hline & Training & Testing & Total \\
\hline Electrons & 10395 & 10405 & 22800 \\
\hline Jets & 3735 & 3774 & 7509 \\
\hline
\end{tabular}

Table 4: Initial organization of the training and testing sets.

\footnotetext{
${ }^{1}$ As an approximation, we assume that the number of independent sources is the same as the number of rings.

${ }^{2}$ For better visualization, we are presenting the generated rings prior to the summing and accumulation process.
} 

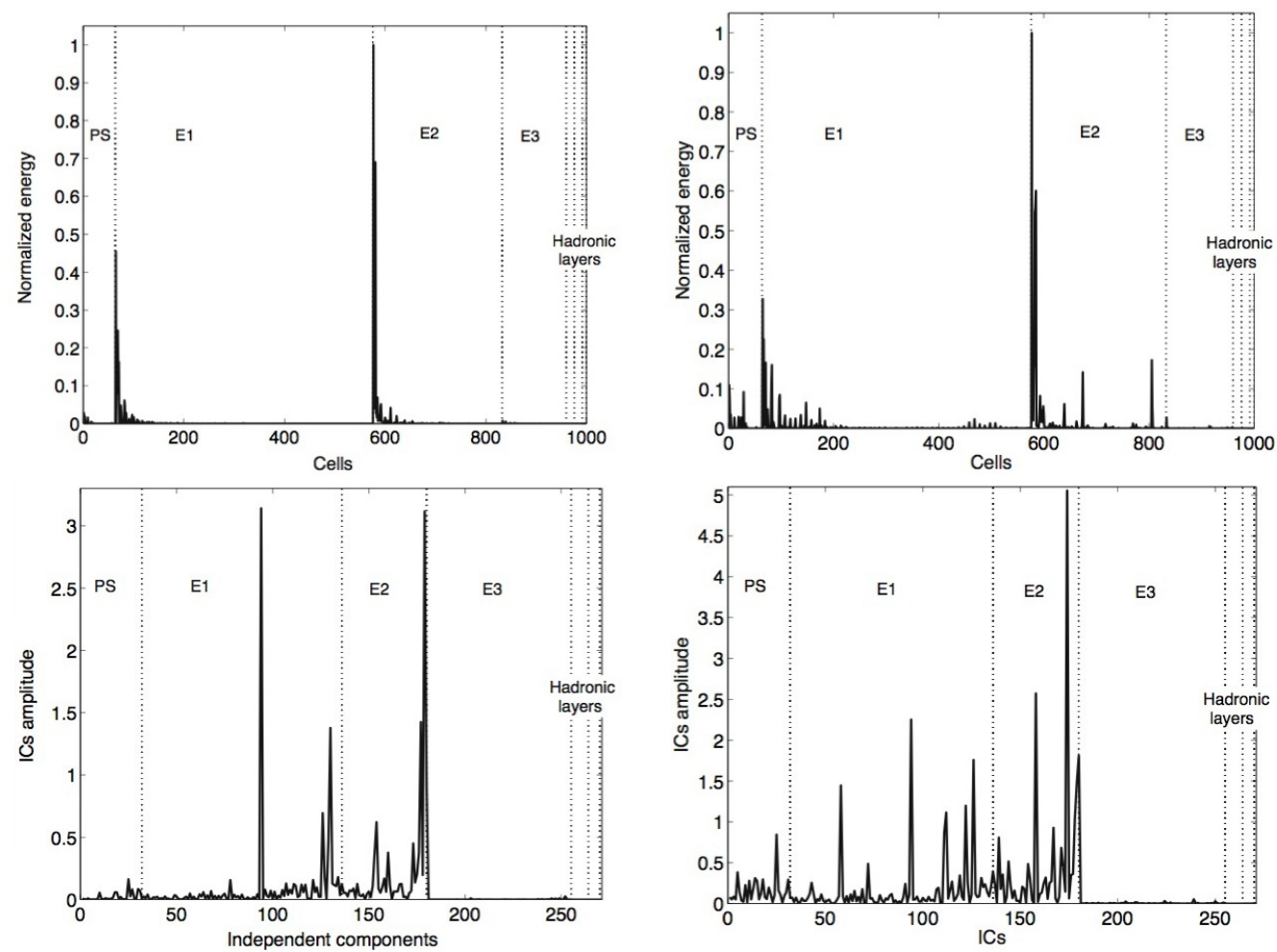

Figure 8: Typical rings for electrons (left) and jets (right) over the EM calorimeter prior to IC projection (top) and after IC projection (bottom).

was the Resilient Backpropagation [14]. The stopping condition was the stabilization of the SP product. The activation function was the hyperbolic tangent for the hidden an output layers. The data sets were initially topologically pre-processed and normalized as describe in Section 4. Then, the training and testing sets were divided as shown in Tab. 4. After that, the training sets were mixed and the ICs were extracted using the FastICA algorithm [11], as described in Section 3. Finally, all sets were projected on these extracted ICs. The training was performed varying the number of hidden nodes from 1 to 5 , and the ROCs obtained from each training can be visualized in Fig 9. As we can observe, the best result was achieved using 4 hidden nodes, resulting in a detection efficiency of $96.4 \%$ for a false alarm of only $3.9 \%$.

For comparison purposes, the efficiency of the T2Calo [15], which is the baseline electron / jet discriminator for the ATLAS collaboration, is also presented in Fig. 9. As one can verify, the proposed discriminator was able to achieve, for the same detection efficiency, almost 7 times more jets rejection than T2Calo. Additionally, we presented the ROC obtained by feeding a 12 hidden nodes neural network just with the ring sums (without IC projection). The result is almost the same as it is achieved using IC and a linear neural network, pointing to the direction that independent components helped to non-linearly uncorrelate the input patterns, in such a way that the hypothesis making section was relieved from such task.

As mentioned in Section 2, the calorimeters have different resolutions within a given layer. The resolution decreases as the $\eta$ coordinate moves toward the edges of the calorimeter. So, in Fig. 10 


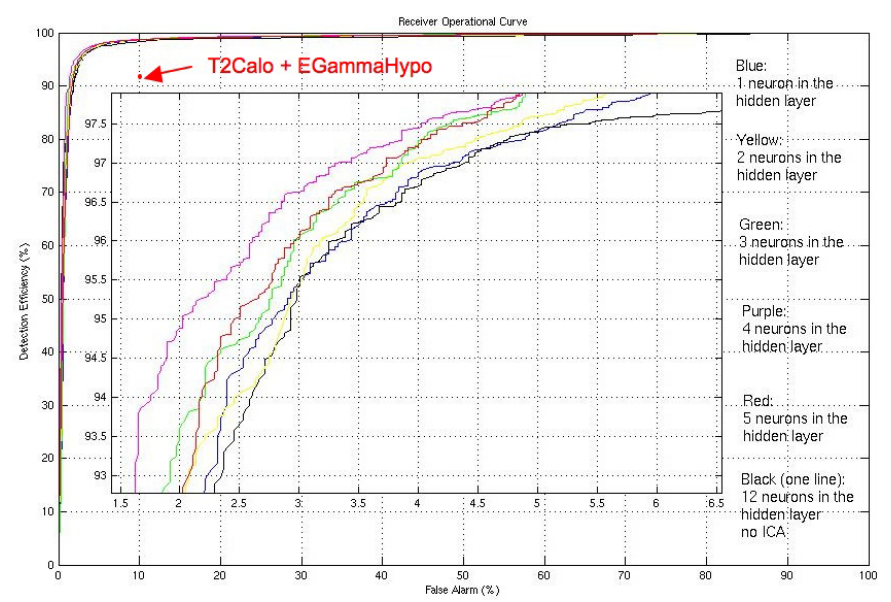

Figure 9: ROC obtained for the discriminator.

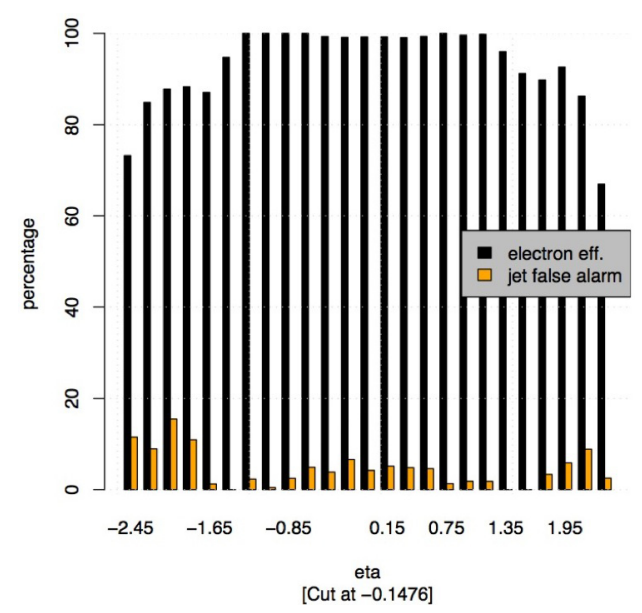

Figure 10: Detection efficiency variation according to $\eta$.

is presented the variation of the detection efficiency according to $\eta$. As expected, the discriminator maintained high detection efficiency until $|\eta|<1.5$, since after that point, the resolution decreases. Also, the lack of sufficient event statistics for this regions contributes for this loss.

Finally, Fig. 11 presents the cumulative timing distributions for each step of the proposed system running in offline mode (outside Athena, which is the framework where the High Level Trigger signal processing algorithms are executed [8]), and considering the RoI information already available (excluding the data retrieval time). The machine used was an Intel Core 2 Duo running at $2.33 \mathrm{GHz}$, with 2 GBytes of memory. In average, $329.93 \pm 50.84 \mu \mathrm{s}$ is taken by the proposed system to generate a result, being suitable for the online operation of the ATLAS second-level trigger. As one can see, the projection onto the independent components implied an overhead of only $24.93 \pm 1.45 \mu \mathrm{s}$, representing less than $10 \%$ of the total algorithm time, resulting in a good trade-off between detection efficiency and execution time. 


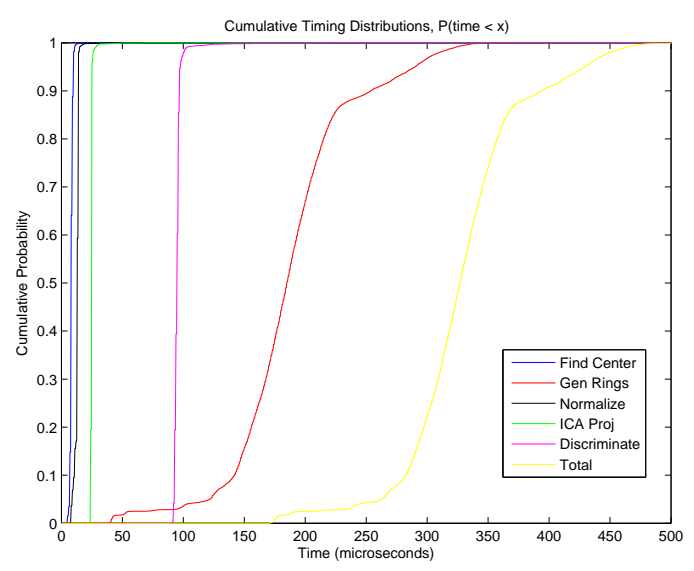

Figure 11: Cumulative timing distribution for each step of the proposed discriminating system.

\section{Conclusions}

This paper presented a second-level electron/jet discriminating system for particle identification in the stringent conditions of LHC, which is the next generation particle collider. To reduce the very high input space dimension, a topological pre-processing method based on ring sums was performed. In addition, by means of independent component analysis, the independent sources from the input signals were extracted and fed the input nodes of a neural network, which performed the particle identification. Using neural processing on independent components, a high detection efficiency (96.4\% electron identification for a false alarm probability as low as $3.9 \%$ ). Timing measurements also proved that the proposed algorithm is able to cope with the stringent time restrictions of the ATLAS second-level trigger.

\section{Acknowledgment}

The authors would like to thanks CAPES, CNPq and FAPERJ (Brazil) and CERN (Switzerland). We would also like to thank our colleagues from the Trigger / DAQ collaboration of the ATLAS detector for providing the simulation data and fruitful discussions concerning this work.

\section{References}

[1] Proceedings of Computing in High Energy and Nuclear Physics, 2006.

[2] G. F. Knoll, Radiation Detection and Measurement. John Wiley \& Sons, 2nd ed., 1989.

[3] CERN, "The large hadron collider project."

[4] CERN, "The ATLAS project."

[5] R. Wigmans, Calorimetry: Energy Measurement In Particle Physics. Oxford, 2000.

[6] L. Peak and K. Varvell, "The physics of the standard model part 1: General and mathematical introduction." 
[7] ATLAS Level-1 Trigger Group, ATLAS level-1 trigger technical design report, report, CERN, ATLAS TDR-12, June, 1998.

[8] ATLAS HLT/DAQ/DCS Group, ATLAS: High-level trigger data aquisition and controls, report, CERN, ATLAS TDR-016, October, 2003.

[9] S. Brown and Z. Vranesic, Fundamentals of Digital Logic with VHDL Design. McGraw-Hill, 2000.

[10] A. Hyvärinen, J. Karhunen, and E. Oja, Independent Component Analysis. John Wiley \& Sons, 2001.

[11] A. Hyvärinen and E. Oja, A fast fixed-point algorithm for independent component analysis, 1997.

[12] A. dos Anjos, M. Abolins, S. Armstrong, J. T. Baines, M. Barisonzi, H. P. Beck, C. P. Bee, M. Beretta, M. Biglietti, and R. Blair, The second level trigger of the atlas experiment at cern's lhc, June, 2004.

[13] A. dos Anjos, R. C. Torres, J. M. Seixas, B. C. Ferreira, and T. C. Xavier, Neural triggering system operating on high resolution calorimetry information, May, 2006.

[14] M. Riedmiller and H. Braun, A direct adaptive method for faster backpropagation learning: The RPROP algorithm, in Proceedings of the IEEE International Conference on Neural Networks, (San Francisco, CA), pp. 586-591, 1993.

[15] The PESA Core Algorithms Group, Algorithms for the ATLAS high level trigger, manual, CERN, June, 2003. 